\title{
Diagnostic accuracy of diffusion-weighted whole-body imaging with background body signal suppression/T2-weighted image fusion for the detection of abdominal solid cancer
}

\author{
MINORU TOMIZAWA ${ }^{1}$, FUMINOBU SHINOZAKI ${ }^{2}$, KAZUNORI FUGO $^{3}$, SATOMI TANAKA $^{2}$, \\ TAKAFUMI SUNAOSHI ${ }^{2}$, DAISUKE KANO ${ }^{2}$, ERIKO SUGIYAMA ${ }^{2}$, MISAKI SHITE $^{2}$, \\ RYOUTA HAGA $^{2}$, YOSHIYA FUKAMIZU ${ }^{2}$, TOSHIYUKI FUJTA ${ }^{2}$, SATOSHI KAGAYAMA ${ }^{2}$, \\ RUMIKO HASEGAWA ${ }^{4}$, AKIRA TOGAWA ${ }^{4}$, YOSHINORI SHIRAI ${ }^{4}$, NOBORU ICHIKI ${ }^{4}$, YUJI OSHIMA ${ }^{5}$, \\ NAOTO KOIKE ${ }^{5}$, YASUKO TOSHIMITSU ${ }^{6}$, YASUFUMI MOTOYOSHI ${ }^{7}$, TAKAO SUGIYAMA ${ }^{8}$, \\ SHIGENORI YAMAMOTO ${ }^{9}$, TAKASHI KISHIMOTO ${ }^{3}$ and NAOKI ISHIGE $^{10}$
}

\begin{abstract}
Departments of ${ }^{1}$ Gastroenterology and ${ }^{2}$ Radiology, National Hospital Organization, Shimoshizu Hospital, Yotsukaido, Chiba 284-0003; ${ }^{3}$ Department of Molecular Pathology, Chiba University Graduate School of Medicine, Chiba, Chiba 260-8670; ${ }^{4}$ Department of Surgery, National Hospital Organization, Shimoshizu Hospital, Yotsukaido, Chiba 284-0003; ${ }^{5}$ Department of Surgery, Seirei Sakura Citizen Hospital, Sakura, Chiba 285-8765; ${ }^{6}$ Department of Surgery, National Hospital Organization Chiba Medical Center, Chiba, Chiba 260-8606; Departments of ${ }^{7}$ Neurology, ${ }^{8}$ Rheumatology, ${ }^{9}$ Pediatrics and ${ }^{10}$ Neurosurgery, National Hospital Organization Shimoshizu Hospital, Yotsukaido, Chiba 284-0003, Japan
\end{abstract}

Received January 17, 2016; Accepted January 13, 2017

DOI: $10.3892 /$ etm.2017.4432

\begin{abstract}
Diffusion-weighted whole-body imaging with background body signal suppression (DWIBS) images show significant contrast for cancer tissues against non-cancerous tissues. Fusion of a DWIBS and a T2-weighted image (DWIBS/T2) can be used to obtain functional, as well as anatomic, information. In the present study, the performance of DWIBS/T2 in the diagnosis of abdominal solid cancer was evaluated. The records of 14 patients were retrospectively analyzed [5 patients with hepatocellular carcinoma (HCC), 4 with metastatic liver cancer, 3 with pancreatic cancer, 1 with renal cellular carcinoma and 1 with malignant lymphoma of the para-aortic lymph node]. T1WI and T2WI scans did not detect pancreatic cancer in certain cases, whereas DWIs and DWIBS/T2 clearly demonstrated pancreatic cancer in all cases. In addition, metastatic liver cancer and HCC were successfully detected with abdominal US and CECT; however, US did not detect pancreatic cancer in 1 case, while CECT and DWIBS/T2 detected pancreatic cancer in all cases. In conclusion, the diagnostic performance of DWIBS/T2 was the same as that of abdominal US and CECT in detecting primary
\end{abstract}

Correspondence to: Dr Minoru Tomizawa, Department of Gastroenterology, National Hospital Organization, Shimoshizu Hospital, 934-5 Shikawatashi, Yotsukaido, Chiba 284-0003, Japan E-mail: nihminor-cib@umin.ac.jp

Key words: hepatocellular carcinoma, pancreatic cancer, abdominal ultrasonography, computed tomography and metastatic liver cancer. DWIBS/T2 enabled the diagnosis of pancreatic cancer in cases where it was not detected with US, T1WI or T2WI.

\section{Introduction}

Diffusion-weighted whole-body imaging with background body signal suppression (DWIBS) is acquired using multiple signal averaging, prepulse fat suppression and heavy diffusion weighting during free breathing (1). This technique is based on diffusion-weighted imaging, which visualizes and assesses the random movement of water at the molecular level (also known as Brownian motion) (2). DWIBS significantly increases the signal-to-noise ratio and is useful for detecting and staging tumors, as well as monitoring the response to therapy $(3,4)$. DWIBS is used to detect cancer in organs that are not influenced by respiration, such as in the breast (5). The existing literature regarding DWIBS mainly involves breast cancer, bone metastasis and malignant lymphoma (6-13). DWIBS is not commonly used to detect abdominal solid cancer, since the anatomical analysis of these tumors can be difficult and respiratory movement makes the image obscure $(14,15)$. Sommer et al (16) reported on the use of DWIBS for detecting liver cancer; however, they mainly used it for the diagnosis of malignant lymphoma and breast cancer.

By overlapping a DWIBS image onto a T2-weighted image (T2WI) using a workstation, fusion of the DWIBS and T2WI (DWIBS/T2) can be performed $(4,16,17)$. DWIBS/T2 clearly illustrates functional information through anatomic images, suggesting that DWIBS/T2 may enable the detection of cancer in the abdominal solid organs. Thus, DWIBS/T2 may be a 
potential diagnostic strategy for the diagnosis of abdominal solid cancer. The present study aimed to retrospectively analyze the performance of DWIBS/T2 in the diagnosis of abdominal solid (not-alimentary) cancer.

\section{Materials and methods}

Ethical statement. The present study was approved by the Ethics Committee of the National Hospital Organization, Shimoshizu Hospital (Yotsukaido, Japan). As procedures were performed as a part of routine clinical practice, written informed consent for inclusion into the study was waived. Patient records were anonymized and retrospectively analyzed. Written informed consent was, however, obtained from all patients who were subjected to contrast-enhanced computed tomography (CECT) and DWIBS/T2, due to an associated risk of adverse side effects. Informed consent was also obtained from patients who were subjected to abdominal ultrasonography (US), though written consent was waived as abdominal US is considered to be a safe and non-invasive therapeutic method (18).

Diagnostic procedure. Patients were subjected to abdominal US and CECT when they were suspected of malignancy in the abdominal cavity. DWIBS/T2 was performed to reveal the extent of the malignancy when the extent was not clear in the abdominal US or CECT images. DWIBS/T2 was also performed to accumulate information of positivity or negativity of the suspected lesion for more accurate diagnosis. It was expected that DWIBS/T2 showed the extent of the malignancy more clearly, due to the strong contrast of positive signals against the surrounding healthy tissues. These results were compared with the histological diagnosis or follow-up.

Inclusion criteria. Patient records, including imaging results, were analyzed retrospectively from patients admitted to National Hospital Organization, Shimoshizu Hospital between November 2012 and May 2014. Patients were enrolled in the present study if they were suspected to have abdominal cancer and had results available from abdominal US, CECT and DWIBS/T2 examinations. For patients referred to another hospital with suspicion of malignancy, the findings of other hospitals were received to confirm our diagnosis. Patients managed in the National Hospital Organization, Shimoshizu Hospital were followed-up with abdominal US, CECT and DWIBS/T2 examinations for up to 11 months.

Exclusion criteria. Patients were excluded from the current study when their abdominal US, CECT or DWIBS/T2 results were not available. In addition, patients referred to other hospitals were excluded when the response of the hospital was not available.

Abdominal US examination. Senior fellows of the Japan Society of Ultrasonics in Medicine (Tokyo, Japan) performed the abdominal US examination with an SSA-700A instrument (Toshiba Medical Systems Corp., Ohtawara, Japan) using a 3.75-MHz curved-array probe (PVT-375BT) (Toshiba Medical Systems Corp.).
CECT examination. CECT was performed using a 16-detector row CT scanner (SOMATOM Emotion 16; Siemens, Munich, Germany). Imaging parameters for three-phase contrast-enhanced images were as follows: Tube voltage, $130 \mathrm{kVp}$; gantry rotation speed, 0.6 rotations/sec; and maximum allowable tube current, $120 \mathrm{~mA}$. Patients were administered the intravenous contrast medium (100 ml iopamidol; Konica Minolta Healthcare, Tokyo, Japan) at $3 \mathrm{ml} / \mathrm{sec}$. CT images were acquired prior to injection of contrast medium and at 30 , 70 , and $180 \mathrm{sec}$ later.

Magnetic resonance imaging (MRI) examination. All MRI studies were performed using a 1.5 Tesla scanner (Achieva software, version 3.2.2; Philips Medical Systems, Best, The Netherlands). The T1-weighted image (T1WI), T2WI and diffusion-weighted image (DWI) were obtained with pulse sequences as depicted in Table I. DWIBS/T2 images were constructed with the Extended MR WorkSpace 2.6.3.4 software (Philips Medical Systems). The DWI gradients were applied along the $\mathrm{x}-, \mathrm{y}$ - and $\mathrm{z}$-axes before and after a $180^{\circ}$ inversion prepulse to obtain fat-saturated, isotropic images with DWI sensitivity using the following parameters for a single stack: b value, 0 and $800 \mathrm{~mm}^{2} / \mathrm{sec}$; TR/TE/IR, 6960/79/150 msec; acquisition matrix, 176x115; reconstruction matrix, 256; field of view, $530 \mathrm{~mm}$ (right/left), $349 \mathrm{~mm}$ (anterior/posterior) and $226 \mathrm{~mm}$ (feet/head); slice thickness, $6 \mathrm{~mm}$; size of reconstructed voxel, $2.07 \times 2.08 \times 6 \mathrm{~mm}^{3}$.

Staging. Staging of cancer tissue was determined according to guidelines of the American Joint Committee on Cancer classification (19). Briefly, the tumor stages assigned in the present study were as follows: For hepatocellular carcinoma (HCC): T2: Solitary tumor with vascular invasion or multiple tumors $\leq 5 \mathrm{~cm}$, T3a: Multiple tumors $>5 \mathrm{~cm}, \mathrm{~T} 3 \mathrm{~b}$ : Single tumor or multiple tumors of any size involving a major branch of the portal vein or hepatic vein. For pancreatic cancer: T3: Tumor extends beyond the pancreas but without involvement of the celiac axis of the superior mesenteric artery, T4: Tumor involves the celiac axis or the superior mesenteric artery. For renal cellular carcinoma: T1b: Tumor size $>4 \mathrm{~cm}$ but not $<7 \mathrm{~cm}$ in greatest dimension and limited to the kidney.

\section{Results}

Patients. A total of 14 patients were enrolled into the current study, including 5 males (mean age, 79.2 \pm 5.8 years) and 9 females (mean age, $73.0 \pm 4.7$ years). The patients were diagnosed with abdominal US, CECT, T1WI, T2WI, DWI and DWIBS/T2. Of the 14 patients, 5 were diagnosed with HCC, 4 with metastatic liver cancer, 3 with pancreatic cancer, 1 with renal cellular carcinoma and 1 with malignant lymphoma of the para-aortic lymph node. No patients suffered from adverse side effects.

Diagnostic performance of DWIBS/T2 compared with T1WI and T2WI. To evaluate the diagnostic performance of DWIBS/T2, the detectability of abdominal cancer was compared among T1WI, T2WI, DWI and DWIBS/T2 (Table II). DWI and DWIBS/T2 indicated positive results in all the patients with a definitive diagnosis of abdominal 
Table I. Pulse sequences in the present study.

\begin{tabular}{lccc}
\hline Parameter & T1-weighted image & T2-weighted image & DWI and DWIBS/T2 \\
\hline Sequence & GRE & Single-shot SE & EPI SE \\
TR (msec) & 182 & 1,000 & 11,250 \\
TE $(\mathrm{msec})$ & First: 2.3 (out phase) & 90 & 83 \\
& Second: 4.6 (in phase) & 90 & 90 \\
Flip angle $\left(^{\circ}\right)$ & 75 & 1 & 4 \\
NSA & 1 & 8 & 5 \\
Slice thickness $(\mathrm{mm})$ & 8 & 1 & 0 \\
Slice gap & 1 & No & SPAIR \\
Fat saturation & No & Posterior-anterior & Posterior-anterior \\
Phase encoding direction & Posterior-anterior & & 5 \\
\hline
\end{tabular}

DWI, diffusion-weighted image; DWIBS, diffusion-weighted whole-body imaging with background body signal suppression/T2-weighted image fusion; GRE, gradient echo; SE, spin echo; EPI, echo planer imaging; TR, time to repetition; TE, time to echo; NSA, number of signal averages.

Table II. Detection of cancer with magnetic resonance imaging.

\begin{tabular}{|c|c|c|c|c|c|c|}
\hline Patient number & Final diagnosis & Stage $^{a}$ & T1WI & $\mathrm{T} 2 \mathrm{WI}$ & DWI & DWIBS/T2 \\
\hline 1 & Hepatocellular carcinoma & $\mathrm{T} 3 \mathrm{a}$ & $(+)$ & $(+)$ & $(+)$ & $(+)$ \\
\hline 2 & Hepatocellular carcinoma & $\mathrm{T} 2$ & $(-)$ & $(-)$ & $(+)$ & $(+)$ \\
\hline 3 & Hepatocellular carcinoma & $\mathrm{T} 3 \mathrm{~b}$ & $(+)$ & $(+)$ & $(+)$ & $(+)$ \\
\hline 4 & Hepatocellular carcinoma & $\mathrm{T} 3 \mathrm{~b}$ & $(+)$ & $(+)$ & $(+)$ & $(+)$ \\
\hline 5 & Hepatocellular carcinoma & $\mathrm{T} 2$ & $(+)$ & $(+)$ & $(+)$ & $(+)$ \\
\hline 6 & Metastatic liver cancer & NA & $(+)$ & $(+)$ & $(+)$ & $(+)$ \\
\hline 7 & Metastatic liver cancer & NA & $(+)$ & $(+)$ & $(+)$ & $(+)$ \\
\hline 8 & Metastatic liver cancer & NA & $(+)$ & $(+)$ & $(+)$ & $(+)$ \\
\hline 9 & Metastatic liver cancer & NA & $(+)$ & $(+)$ & $(+)$ & $(+)$ \\
\hline 10 & Pancreatic cancer & $\mathrm{T} 3$ & $(+)$ & $(+)$ & $(+)$ & $(+)$ \\
\hline 11 & Pancreatic cancer & $\mathrm{T} 4$ & $(-)^{\mathrm{b}}$ & $(-)^{\mathrm{b}}$ & $(+)$ & $(+)$ \\
\hline 12 & Pancreatic cancer & $\mathrm{T} 3$ & $(-)$ & $(-)$ & $(+)$ & $(+)$ \\
\hline 13 & Renal cell carcinoma & $\mathrm{T} 1 \mathrm{~b}$ & $(+)$ & $(+)$ & $(+)$ & $(+)$ \\
\hline 14 & Malignant lymphoma $^{c}$ & NA & $(+)$ & $(+)$ & $(+)$ & $(+)$ \\
\hline
\end{tabular}

${ }^{\mathrm{a} A m e r i c a n}$ joint committee on cancer classification (7th edition). ${ }^{\mathrm{b}} \mathrm{Head}$ of pancreas slightly swollen without any difference in the intensity compared with the surrounding tissue. ${ }^{\circ}$ Originating from the para-aortic lymph node. T1WI, T1-weighted image; T2WI, T2-weighted image; DWI, diffusion-weighed image; DWIBS/T2, diffusion-weighted whole-body imaging with background body signal suppression T2-weighted image fusion; (+), positive results; (-), negative results; NA, not applicable.

cancer. In addition, the results of DWI were in accordance with those of DWIBS/T2. These results suggested that DWI and DWIBS/T2 had the same sensitivity of the detection of cancer. By contrast, T1WI and T2WI scans indicated negative results in 1 patient with hepatocellular carcinoma (HCC) and in 2 patients with pancreatic cancer. These results suggested that DWI and DWIBS/T2 were more sensitive than T1WI and T2WI in the detection of cancer.

Fig. 1 shows the representative case of a 73-year-old female who visited the National Hospital Organization, Shimoshizu Hospital with general malaise. HCC was detected in this patient as a space-occupying lesion with mixed enhancement using
CECT (Fig. 1A). However, T1WI (Fig. 1B) or T2WI (Fig. 1C) scans were negative for HCC. The lesion was clearly observed as a high signal in the DWIBS/T2 image (Fig. 1D). The lesion was finally diagnosed as HCC with ultrasound-guided fine needle biopsy. These findings suggested that DWIBS/T2 was more useful for the detection of HCC than T1WI and T2WI. Written informed consent was obtained from this patient prior to the use of her data in the present study.

Diagnostic performance of DWIBS/T2 compared with US and CECT. To evaluate ability of DWIBS/T2 to detect cancer, images were compared with abdominal US and CECT 
Table III. Comparison of abdominal US, CECT and DWIBS/T2 findings.

\begin{tabular}{|c|c|c|c|c|c|}
\hline Patient number & Final diagnosis & Stage $^{a}$ & Abdominal US & CECT & DWIBS/T2 \\
\hline 1 & Hepatocellular carcinoma & T3a & $(+)$ & $(+)$ & $(+)$ \\
\hline 2 & Hepatocellular carcinoma & $\mathrm{T} 2$ & $(+)$ & $(+)$ & $(+)$ \\
\hline 3 & Hepatocellular carcinoma & $\mathrm{T} 3 \mathrm{~b}$ & $(+)$ & $(+)$ & $(+)$ \\
\hline 4 & Hepatocellular carcinoma & T3b & $(+)$ & $(+)$ & $(+)$ \\
\hline 5 & Hepatocellular carcinoma & $\mathrm{T} 2$ & $(+)$ & $(+)$ & $(+)$ \\
\hline 6 & Metastatic liver cancer & NA & $(+)$ & $(+)$ & $(+)$ \\
\hline 7 & Metastatic liver cancer & NA & $(+)$ & $(+)$ & $(+)$ \\
\hline 8 & Metastatic liver cancer & NA & $(+)$ & $(+)$ & $(+)$ \\
\hline 9 & Metastatic liver cancer & NA & $(+)$ & $(+)$ & $(+)$ \\
\hline 10 & Pancreatic cancer & T3 & $(+)$ & $(+)$ & $(+)$ \\
\hline 11 & Pancreatic cancer & $\mathrm{T} 4$ & $(+)$ & $(+)$ & $(+)$ \\
\hline 12 & Pancreatic cancer & T3 & $(-)$ & $(+)$ & $(+)$ \\
\hline 13 & Renal cell carcinoma & T1b & $(+)$ & $(+)$ & $(+)$ \\
\hline 14 & Malignant lymphoma & NA & $(+)$ & $(+)$ & $(+)$ \\
\hline
\end{tabular}

${ }^{a}$ American joint committee on cancer classification (7th edition). US, abdominal ultrasound; CECT, contrast-enhanced computed tomography; DWIBS/T2, diffusion-weighted whole-body imaging with background body signal suppression T2-weighted image fusion; (+), positive results; $(-)$, negative results; NA, not applicable.
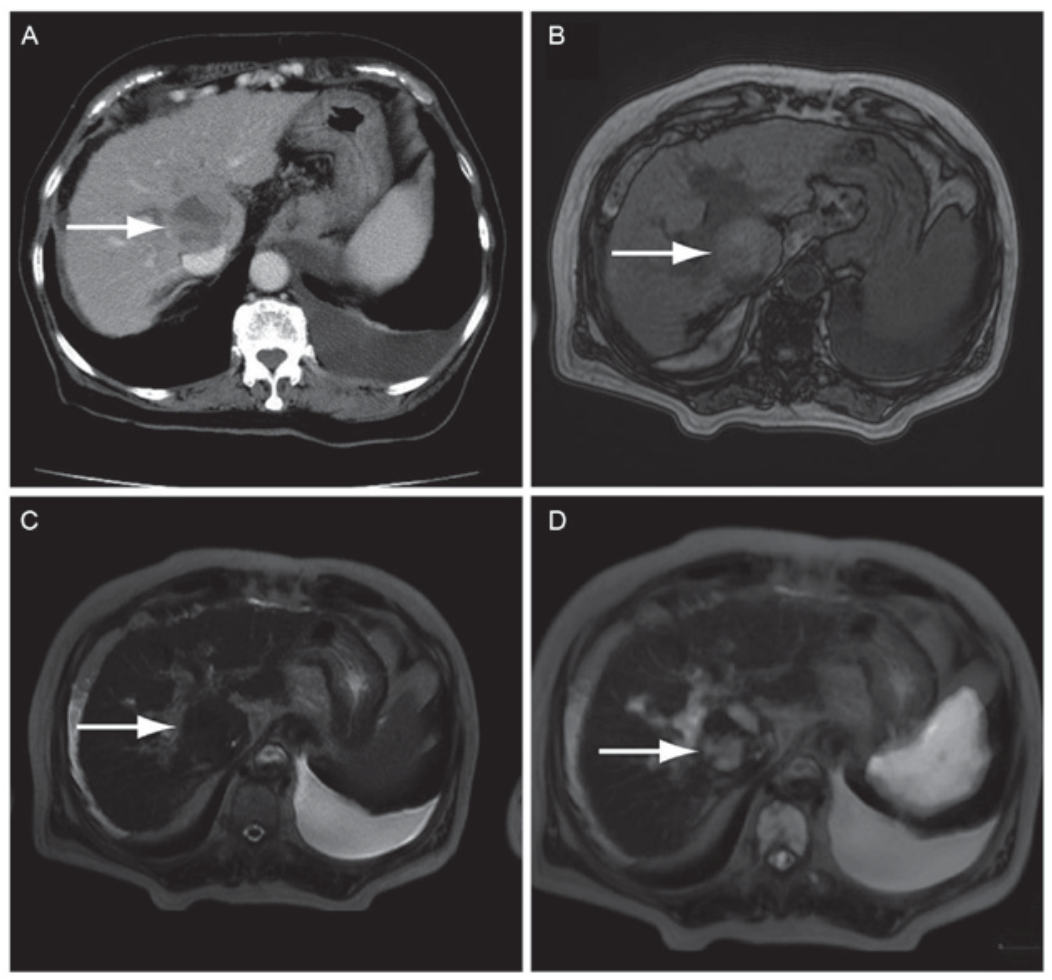

Figure 1. Imaging examination in the representative case of a 73-year-old patient diagnosed with hepatocellular carcinoma. The tumor was successfully detected with DWIBS/T2. (A) CECT scanning demonstrated a space-occupying lesion with mixed enhancement (arrow). (B) T1WI showed an unclear mass-like lesion. (C) Detection of the lesion was difficult in a T2WI when compared with the CECT scan. (D) DWIBS/T2 clearly showed a high signal. DWIBS/T2, diffusion-weighted whole-body imaging with background body signal suppression/T2-weighted image fusion; CECT, contrast-enhanced computed tomography; WI, weighted image.

(Table III). The detectability of HCC and metastatic liver cancer using DWIBS/T2 was the same as that of abdominal US and CECT, with the exception of 1 patient with pancreatic cancer who was not correctly diagnosed by abdominal US.
These results suggested that DWIBS/T2 had the same sensitivity as CECT. It was also suggested that DWIBS/T2 was more sensitive when compared with US for the detection of pancreatic cancer. 

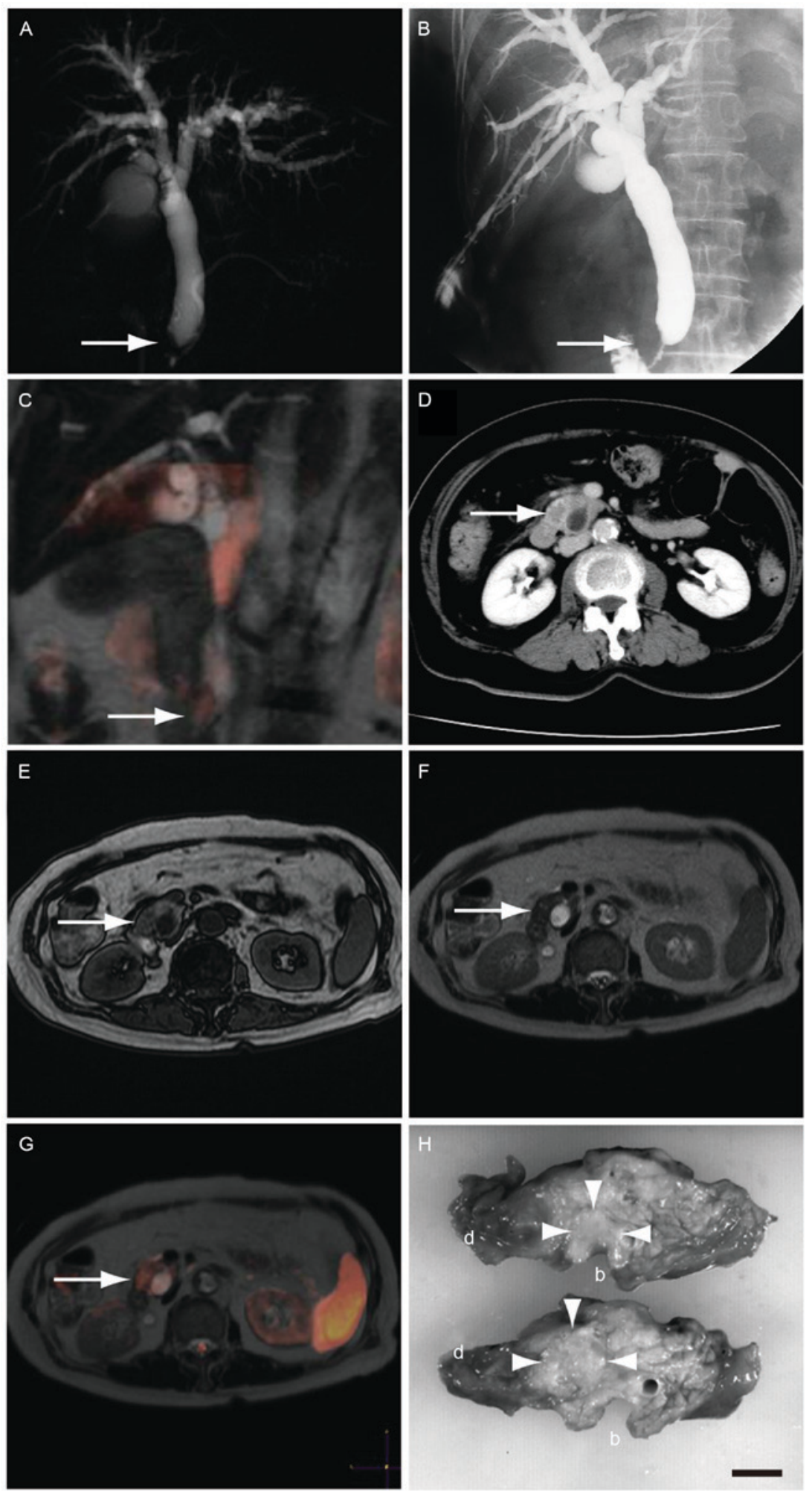

Figure 2. Imaging examination in the representative case of a 75-year-old female patient diagnosed with pancreatic cancer. The tumor was successfully detected by DWIBS/T2. (A) Magnetic resonance cholangiopancreatography showed obstruction of the common bile duct near the papilla of Vater (arrow). (B) Percutaneous transhepatic biliary drainage was performed. (C) A coronal section of DWIBS/T2 demonstrated a high signal on the obstruction. (D) CECT revealed an irregularly shaped low-density area in the head of the pancreas. (E) T1WI and (F) T2WI scans did not show clear presence of pancreatic cancer. (G) A transverse section of DWIBS/T2 demonstrated a high signal in the head of the pancreas. (H) Surgical specimens confirmed the diagnosis of pancreatic cancer in the head of the pancreas close to common bile duct (circled with arrowheads). Scale bar, $1 \mathrm{~cm} ; \mathrm{b}$, common bile duct; d, duodenum; DWIBS/T2, diffusion-weighted whole-body imaging with background body signal suppression/T2-weighted image fusion; CECT, contrast-enhanced computed tomography; WI, weighted image.

Fig. 2 demonstrates the representative case of a 75 -year-old female who visited our hospital with jaundice, and was diagnosed with pancreatic cancer. Cancer in the head of the pancreas was shown as an obstruction of the common bile duct with magnetic resonance cholangiopancreatography (Fig. 2A). In order to reduce jaundice, percutaneous transhepatic biliary 
drainage was performed (Fig. 2B). However, these images did not suggest the existence of pancreatic cancer. A coronal section of DWIBS/T2 examination clearly demonstrated a high signal in the head of pancreas (Fig. 2C), suggesting the presence of pancreatic cancer. In addition, CECT examination revealed a small unclear low density area (Fig. 2D). However, pancreatic cancer was not clear using T1WI (Fig. 2E) or T2WI (Fig. 2F). A high signal was shown in the head of pancreas with a transverse section of DWIBS/T2 (Fig. 2G). The diagnosis of pancreatic cancer was confirmed with a surgical specimen (Fig. 2H).

The aforementioned findings revealed that CECT detected pancreatic cancer, however with reduced contrast to the surrounding tissues when compared with DWIBS/T2. US failed to detect pancreatic cancer in 1 case, possibly due to the location of the cancer at the head of the pancreas, where it was surrounded by gas of the stomach and duodenum. These results suggested that DWIBS/T2 may be useful for the diagnosis of pancreatic cancer.

\section{Discussion}

CECT, T1WI, T2WI and DWI examinations have been demonstrated to have similar diagnostic performance for HCC (20). Among MRI sequences, no difference was observed for the detection of liver malignancy, including HCC and metastatic liver cancer $(21,22)$. In the present study, DWI and DWIBS/T2 examination detected liver malignancy by differentiating the cancer as a region of strong contrast against the surrounding tissues. However, DWIBS/T2 images provided added anatomic information compared with DIW. Thus, the present study further investigated the use of DWIBS/T2 in the detection of abdominal cancer. The results indicated no difference in the diagnostic performance of CECT and DWIBS/T2 for liver malignancy. In addition, a previous study indicated that the diagnostic performance of contrast-enhanced MRI and non-contrast-enhanced MRI does not differ for liver malignancy (23). These previous findings along with the present study results suggest that DWBS/T2 may be more beneficial in the diagnosis of liver malignancy compared with CECT and contrast-enhanced MRI. DWIBS/T2 did not require contrast medium, and may avoid complications of CECT and MRI, including anaphylaxis (22).

US, CECT and MRI are used in the diagnosis of pancreatic cancer in routine clinical practice (24). Endoscopic US is essential for the diagnosis of pancreatic cancer with fine-needle aspiration (25). In addition, US is used as a screening method for pancreatic cancer; however, it is limited by field-of-view restriction, due to the presence of gas in the stomach and intestine (26). Therefore, US can fail to detect pancreatic cancer when the tumor is small or surrounded by gas. Diagnosis of pancreatic cancer by CECT may also be difficult due to insufficient contrast. In the present study, DWI and DWIBS/T2 clearly differentiated malignant tissues from the surrounding tissues, allowing easy detection. Furthermore, DWI and DWIBS/T2 did not require contrast enhancement. DWIBS/T2 provided additional anatomic information to DWI that is useful for the detection of pancreatic cancer (27). These results suggested that DWBS/T2 should be preferably performed prior to CECT examination.
The limitation of the present study was that it included a small number of patients. A larger sample size is required to confirm the results. HCC and pancreatic cancer should be diagnosed when the tumors are small for curative treatment $(28,29)$. Thus, studies on the performance of DWIBS/T2 in detecting early-stage HCC and pancreatic cancer are needed.

In conclusion, the diagnostic performance of DWIBS/T2 was similar to that of US and CECT for the detection of primary and metastatic liver cancer. DWIBS/T2 enabled the diagnosis of pancreatic cancer, which was not detected by US, T1WI or T2WI examination. The findings suggest that DWIBS/T2 may be useful in the diagnosis of early-stage HCC and pancreatic cancer.

\section{References}

1. Takahara T, Imai Y, Yamashita T, Yasuda S, Nasu S and Van Cauteren M: Diffusion weighted whole body imaging with background body signal suppression (DWIBS): Technical improvement using free breathing, STIR and high resolution 3D display. Radiat Med 22: 275-282, 2004.

2. Sehy JV, Ackerman JJ and Neil JJ: Apparent diffusion of water, ions and small molecules in the Xenopus oocyte is consistent with Brownian displacement. Magn Reson Med 48: 42-51, 2002.

3. Attariwala R and Picker W: Whole body MRI: Improved lesion detection and characterization with diffusion weighted techniques. J Magn Reson Imaging 38: 253-268, 2013.

4. Kwee TC, Takahara T, Ochiai R, Nievelstein RA and Luijten PR: Diffusion-weighted whole-body imaging with background body signal suppression (DWIBS): Features and potential applications in oncology. Eur Radiol 18: 1937-1952, 2008.

5. Moschetta M, Telegrafo M, Rella L, Capolongo A, Stabile Ianora AA and Angelelli G: MR evaluation of breast lesions obtained by diffusion-weighted imaging with background body signal suppression (DWIBS) and correlations with histological findings. Magn Reson Imaging 32: 605-609, 2014.

6. Stadlbauer A, Bernt R, Gruber S, Bogner W, Pinker K, van der Riet W, Haller J and Salomonowitz E: Diffusion-weighted MR imaging with background body signal suppression (DWIBS) for the diagnosis of malignant and benign breast lesions. Eur Radiol 19: 2349-2356, 2009.

7. Mürtz P, Krautmacher C, Träber F, Gieseke J, Schild HH and Willinek WA: Diffusion-weighted whole-body MR imaging with background body signal suppression: A feasibility study at 3.0 Tesla. Eur Radiol 17: 3031-3037, 2007.

8. Sakurai Y, Kawai H, Iwano S, Ito S, Ogawa H and Naganawa S: Supplemental value of diffusion-weighted whole-body imaging with background body signal suppression (DWIBS) technique to whole-body magnetic resonance imaging in detection of bone metastases from thyroid cancer. J Med Imaging Radiat Oncol 57: 297-305, 2013.

9. Gandage S, Kachewar S, Aironi V and Nagapurkar A: A comparative study of whole body DWIBS MRI versus bone scan for evaluating skeletal metastases. Australas Med J 5: 619-622, 2012.

10. Akay S, Kocaoglu M, Emer O, Battal B and Arslan N: Diagnostic accuracy of whole-body diffusion-weighted magnetic resonance imaging with $3.0 \mathrm{~T}$ in detection of primary and metastatic neoplasms. J Med Imaging Radiat Oncol 57: 274-282, 2013.

11. Yu SP, He L, Liu B, Zhuang XZ, Liu MJ and Hu XS: Differential diagnosis of metastasis from non-metastatic lymph nodes in cervical cancers: Pilot study of diffusion weighted imaging with background suppression at 3T magnetic resonance. Chin Med J (Engl) 123: 2820-2824, 2010

12. Stecco A, Romano G, Negru M, Volpe D, Saponaro A, Costantino S, Sacchetti G, Inglese E, Alabiso O and Carriero A: Whole-body diffusion-weighted magnetic resonance imaging in the staging of oncological patients: Comparison with positron emission tomography computed tomography (PET-CT) in a pilot study. Radiol Med 114: 1-17, 2009.

13. Koike N, Cho A, Nasu K, Seto K, Nagaya S, Ohshima Y and Ohkohchi N: Role of diffusion-weighted magnetic resonance imaging in the differential diagnosis of focal hepatic lesions. World J Gastroenterol 15: 5805-5812, 2009. 
14. Ohno Y, Koyama H, Onishi Y, Takenaka D, Nogami M, Yoshikawa T, Matsumoto S, Kotani Y and Sugimura K: Non-small cell lung cancer: Whole-body MR examination for M-stage assessment-utility for whole-body diffusion-weighted imaging compared with integrated FDG PET/CT. Radiology 248 643-654, 2008.

15. Fischer MA, Nanz D, Hany T, Reiner CS, Stolzmann P, Donati OF, Breitenstein S, Schneider P, Weishaupt D, von Schulthess GK and Scheffel H: Diagnostic accuracy of whole-body MRI/DWI image fusion for detection of malignant tumours: A comparison with PET/CT. Eur Radiol 21: 246-255, 2011.

16. Sommer G Wiese M, Winter L, Lenz C, Klarhöfer M, Forrer F, Lardinois D and Bremerich J: Preoperative staging of non-small-cell lung cancer: Comparison of whole-body diffusion-weighted magnetic resonance imaging and 18F-fluorodeoxyglucose-positron emission tomography/ computed tomography. Eur Radiol 22: 2859-2867, 2012.

17. Nechifor-Boilă IA, Bancu S, Buruian M, Charlot M, Decaussin-Petrucci M, Krauth JS, Nechifor-Boilă AC and Borda A: Diffusion weighted imaging with background body signal suppression/T2 image fusion in magnetic resonance mammography for breast cancer diagnosis. Chirurgia (Bucur) 108: 199-205, 2013.

18. Genc A, Ryk M, Suwala M, Żurakowska T and Kosiak W: Ultrasound imaging in the general practitioner's office-a literature review. J Ultrason 16: 78-86, 2016.

19. Rutkowski P, Wozniak A, Debiec-Rychter M, Kąkol M, Dziewirski W, Zdzienicki M, Ptaszynski K, Jurkowska M, Limon J and Siedlecki JA: Clinical utility of the new American joint committee on cancer staging system for gastrointestinal stromal tumors: Current overall survival after primary tumor resection. Cancer 117: 4916-4924, 2011.

20. Chen HY, Hou YL, Ma XM, Xie HY, Ye M and Bai YR: CT and MRI in target delineation in primary hepatocellular carcinoma. Cancer Radiother 17: 750-754, 2013.
21. Kim YK, Kim YK, Park HJ, Park MJ, Lee WJ and Choi D: Noncontrast MRI with diffusion-weighted imaging as the sole imaging modality for detecting liver malignancy in patients with high risk for hepatocellular carcinoma. Magn Reson Imaging 32: 610-618, 2014.

22. Rose TA Jr and Choi JW: Intravenous imaging contrast media complications: The basics that every clinician needs to know. Am J Med 128: 943-949, 2015

23. Kim YK, Kim YK, Park HJ, Park MJ, Lee WJ and Choi D: Noncontrast MRI with diffusion-weighted imaging as the sole imaging modality for detecting liver malignancy in patients with high risk for hepatocellular carcinoma. Magn Reson Imaging 32: 610-618, 2014

24. He J, Page AJ, Weiss M, Wolfgang CL, Herman JM and Pawlik TM: Management of borderline and locally advanced pancreatic cancer: Where do we stand? World J Gastroenterol 20: 2255-2266, 2014.

25. Guo X, Cui Z and Hu Z: Role of endoscopic ultrasound in treatment of pancreatic cancer. Endosc Ultrasound 2: 181-189, 2013.

26. Benson MD and Gandhi MR: Ultrasound of the hepatobiliary-pancreatic system. World J Surg 24: 166-170, 2000.

27. Ichikawa T, Erturk SM, Motosugi U, Sou H, Iino H, Araki T and Fujii H: High-b value diffusion-weighted MRI for detecting pancreatic adenocarcinoma: Preliminary results. AJR Am J Roentgenol 188: 409-414, 2007.

28. Minami Y, Nishida N and Kudo M: Therapeutic response assessment of RFA for HCC: Contrast-enhanced US, CT and MRI. World J Gastroenterol 20: 4160-4166, 2014.

29. Bockhorn M, Uzunoglu FG, Adham M, Imrie C, Milicevic M, Sandberg AA, Asbun HJ, Bassi C, Büchler M, Charnley RM, et al: Borderline resectable pancreatic cancer: A consensus statement by the international study group of pancreatic surgery (ISGPS). Surgery 155: 977-988, 2014. 